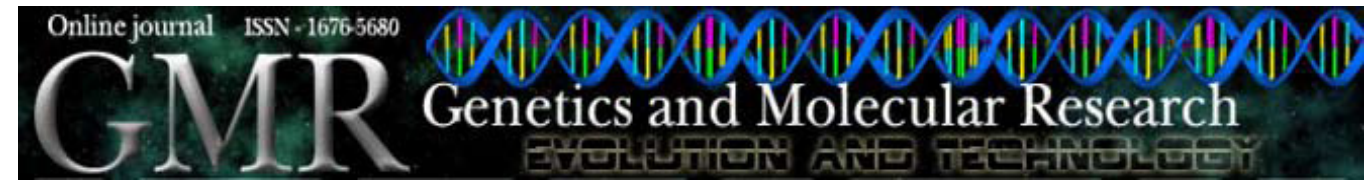

\title{
Interpretation of electrophoretograms of seven microsatellite loci to determine the genetic diversity of the Arabian Oryx
}

\author{
I.A. Arif, H.A. Khan, M. Shobrak, A.A. Al Homaidan, M. Al Sadoon, \\ A.H. Al Farhan and A.H. Bahkali
}

Molecular Fingerprinting and Biodiversity Unit, Prince Sultan Research Chair for Environment and Wildlife, College of Sciences, King Saud University, Riyadh, Saudi Arabia

Corresponding author: H.A. Khan

E-mail:khan_haseeb@yahoo.com

Genet. Mol. Res. 9 (1): 259-265 (2010)

Received October 30, 2009

Accepted January 3, 2010

Published February 9, 2010

\begin{abstract}
Microsatellite markers are commonly used for examining population structure, especially inbreeding, outbreeding and gene flow. An array of microsatellite loci, preferably with multiallelic presentation, is preferable for ensuring accurate results. However, artifact peaks or stutters in the electrophoretograms significantly hamper the reliable interpretation of genotypes. We interpreted electrophoretograms of seven microsatellite loci to determine the genetic diversity of the Arabian Oryx. All the alleles of different loci exhibited good peak resolutions and hence were clearly identified. Moreover, none of the stutter peaks impaired the recognition or differentiation between homozygote and heterozygote. Our findings suggest that correct identification of alleles in the presence of co-amplified nonspecific fragments is important for reliable interpretation of microsatellite data.
\end{abstract}

Key words: Microsatellites; Electrophoretograms; Stutter peaks; Molecular markers; Genetic diversity 


\section{INTRODUCTION}

Microsatellites or simple sequence repeats are widely used markers for molecular fingerprinting. Microsatellites are short sections of DNA where a simple motif, generally 1-5 bp long is repeated up to about 100 times. Microsatellite markers are highly polymorphic, abundant and fairly evenly distributed throughout eukaryotic genomes. The popularity of these markers is due to their ease of amplification by polymerase chain reaction (PCR), their co-dominant nature and their typically high levels of allelic diversity at different loci. There are numerous reports suggesting the usefulness of microsatellite markers for measuring the genetic variability in a wider taxonomic range (Ryberg et al., 2002; Li et al., 2007; Kawka et al., 2007; Chan et al., 2008; Banhos et al., 2008). Although the wet lab analysis of microsatellite markers is quite robust and straightforward, it is often difficult to identify the correct peaks due to appearance of nonspecific peaks in the vicinity of the main allele peak. A genotyping error rate of even $1 \%$, which is an uncommonly good value for most studies, can lead to a substantial number of incorrect multilocus genotypes in a large data set (Hoffman and Amos, 2005). Sources of error include poor amplification, misinterpretation of an artifact peak/band as a true microsatellite allele, incorrect interpretation of stutter patterns or artifact peaks, contamination, mislabeling or data entry errors (Bonin et al., 2004). In many cases, knowing the sources of error in the genotype data can allow one to correct for it, such as re-genotyping homozygous individuals to catch poorly amplifying alleles (Selkoe and Toonen, 2006). However, the most important source of error in microsatellite analysis is probably the incorrect calling of alleles on fluorescent profiles (electrophoretograms) or autoradiographs (Hoffman and Amos, 2005). This report presents the interpretation of 7 microsatellite loci using their respective electrophoretogram images.

\section{MATERIAL AND METHODS}

DNA was extracted from a $200-\mu \mathrm{L}$ blood sample of Arabian Oryx using DNeasy Blood and Tissue Kit (Qiagen GmbH, Germany) according to manufacturer instructions. Seven microsatellite loci including RBP3, BM3501, MCM38, MNS64, MB066, IOBT395, and MCMAI were amplified using earlier reported protocols (MacHugh et al., 1997; Zhou et al., 2007). The primer sequences of these markers are given in Table 1. The forward primer for each marker was labeled with FAM, whereas the reverse primer was unlabelled.

\begin{tabular}{|c|c|c|c|}
\hline Locus & Primer direction & Primer sequence & Annealing temperature $\left({ }^{\circ} \mathrm{C}\right)$ \\
\hline \multirow[t]{2}{*}{ RBP3 } & Forward & 5-TGTATGATCACCTTCTATGCTTC & 55 \\
\hline & Reverse & 5-GCTTTAGGTAATCATCAGATAGC & \\
\hline \multirow[t]{2}{*}{ BM3501 } & Forward & 5-CCAACGGGTTAAAAGCACTG & 58 \\
\hline & Reverse & 5-ТTCCTGTTCCTTCCTCATCTG & \\
\hline \multirow[t]{2}{*}{ MCM38 } & Forward & 5-TGGTGAATGGTGCTCTCATACCAG & 58 \\
\hline & Reverse & 5-CAGCCAGCAGCCTCTAAAGGAC & \\
\hline \multirow[t]{2}{*}{ MNS64 } & Forward & 5-ATTAACTTTGTGGCATCTGAGC & 58 \\
\hline & Reverse & 5-CGTATCAACTAACACGATGCTG & \\
\hline \multirow[t]{2}{*}{ MB066 } & Forward & 5-ATCTGCCTGAAGCCAGTCAC & 58 \\
\hline & Reverse & 5-GGTTTCCTGCACCTGCATGA & \\
\hline \multirow[t]{2}{*}{ IOBT395 } & Forward & 5-ACAACAGGAAAGCTCTGCCA & 58 \\
\hline & Reverse & 5-ACATGTAGCTGTTGATACAGAT & \\
\hline \multirow[t]{2}{*}{ MCMAI } & Forward & 5-CATTACAGCCTGTGTGAGTGTG & 54 \\
\hline & Reverse & 5-GATAGTTCTATCCAACCGTCCC & \\
\hline
\end{tabular}


PCR were performed in a total volume of $20 \mu \mathrm{L}$ containing $2 \mu \mathrm{L} 10 \mathrm{X}$ PCR buffer, $2.5 \mathrm{mM} \mathrm{MgCl}_{2}, 200 \mu \mathrm{M}$ each dNTP, $25 \mathrm{nM}$ of each primer, $25 \mathrm{ng}$ template DNA and $0.5 \mathrm{U}$ Taq DNA polymerase. After initial denaturation at $94^{\circ} \mathrm{C}$ for $4 \mathrm{~min}, 25$ cycles of $93^{\circ} \mathrm{C}$ for $45 \mathrm{~s}, 55^{\circ} \mathrm{C}$ for $45 \mathrm{~s}$ and $72^{\circ} \mathrm{C}$ for $45 \mathrm{~s}$ were repeated followed by the final extension at $72^{\circ} \mathrm{C}$ for $4 \mathrm{~min}$. The aliquots of PCR products $(0.25 \mu \mathrm{L})$ were mixed with 9.25 $\mu \mathrm{L}$ formamide and $0.25 \mu \mathrm{L}$ ROX-500 standard. The contents were heated at $95^{\circ} \mathrm{C}$ for 2 min and then rapidly cooled on ice before being electrophoresed on an 3130XL genetic analyzer. The sizes of electrophoresed DNA fragments (alleles) were determined by the Gene Mapper Software.

\section{RESULTS AND DISCUSSION}

The numbers of alleles for different microsatellite loci observed in this study are as follows: RBP3 (140 and $142 \mathrm{bp}$ ); BM3501 (168 and $170 \mathrm{bp}$ ); MCM38 (108, 110 and $120 \mathrm{bp}$ ); MNS64 (188, 198 and 200 bp); MB066 (128, 130 and 132 bp); IOBT395 (90, 106, 110, and 174); MCMAI (185, 187, 189, and 191). The photos of electrophoretograms for the 7 loci are given in Figures 1-7, respectively, and the interpretation of allele peaks is described in the each figure caption accordingly. All the alleles of different loci exhibited good peak resolutions and hence were clearly identified. Moreover, none of the stutter peaks impaired the recognition or differentiation between homozygote and heterozygote.
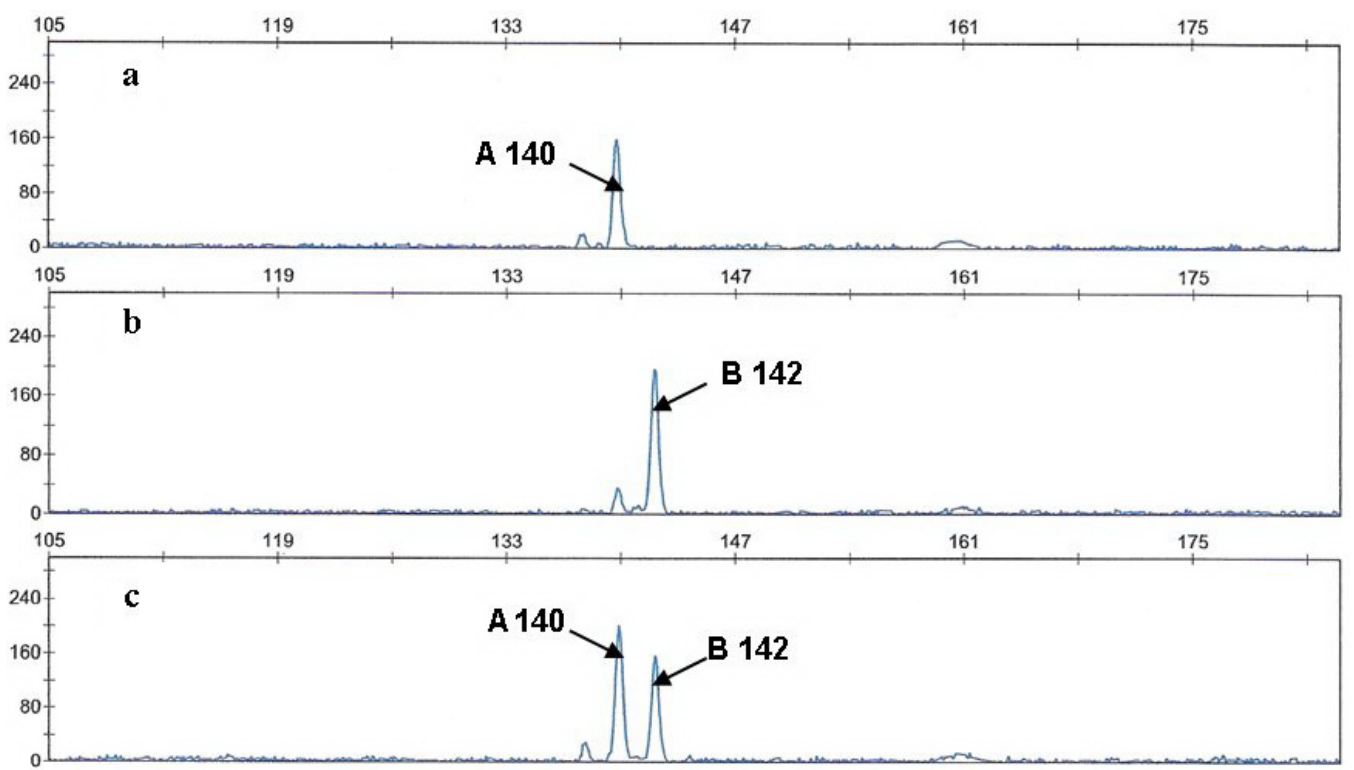

Figure 1. RBP3 locus with 2 alleles, A (140 bp) and B (142 bp). Both the alleles have sharp peaks and contain only a single stutter of allele size $-2 \mathrm{bp}$, respectively. The homozygous presentation of alleles A and B is shown in (a) and (b), respectively. In the case of heterozygote (c), the peak of allele A is slightly higher than the peak of allele B due to the additive effect of the stutter of allele B. 

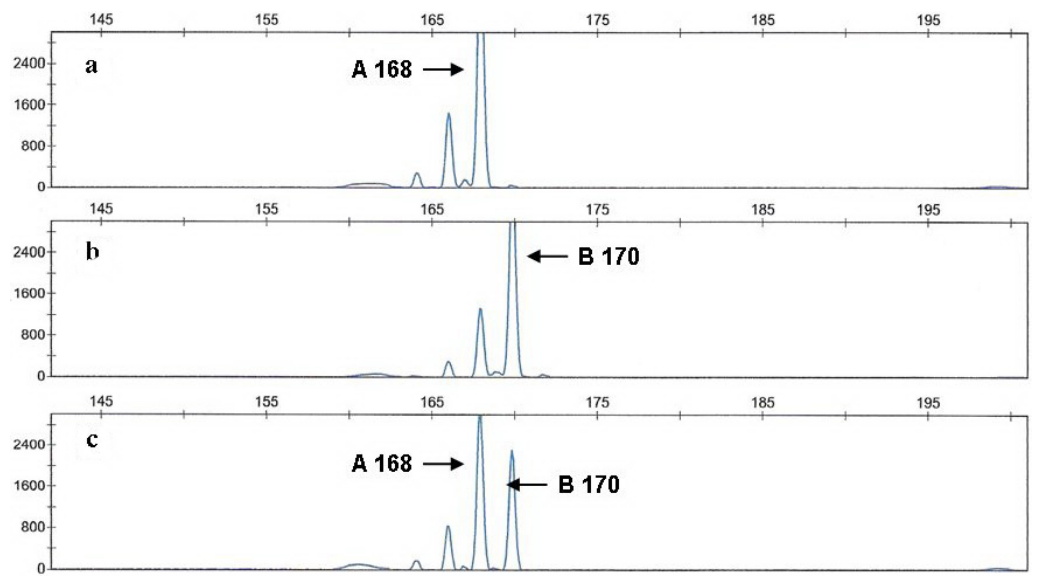

Figure 2. BM3501 locus with 2 alleles, A (168 bp) and B (170 bp). Both the alleles have sharp peaks but also contain three stutters each, 1, 2 and $4 \mathrm{bp}$ shorter than the main alleles. The homozygous presentation of alleles A and B is shown in (a) and (b), respectively. In the case of heterozygote (c), the peak of allele A is slightly higher than the peak of allele B due to the additive effect of the "-2" stutter of allele B.

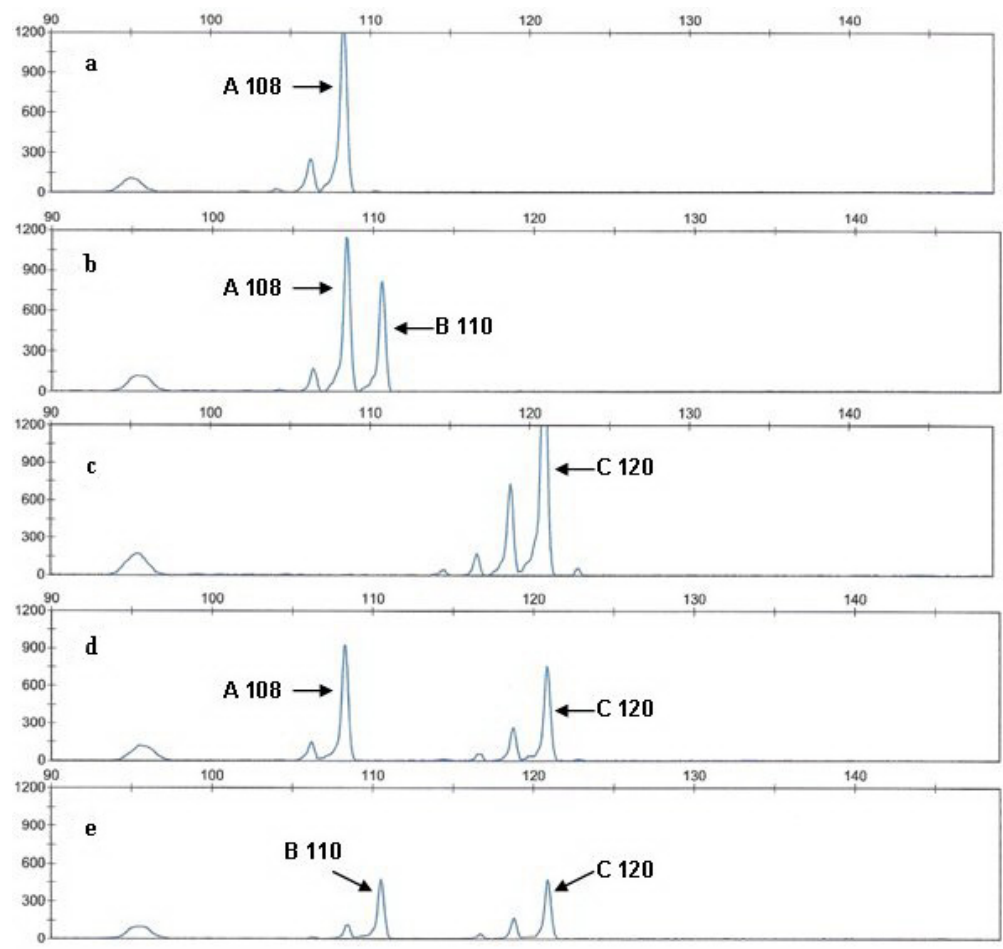

Figure 3. MCM38 locus with 3 alleles, A (108 bp), B (110 bp) and C (120 bp). All the alleles show well-resolved peaks. Alleles A and B have 1 stutter peak 2 bp shorter than the main allele. Allele C contains 2 stutters, 2 and $4 \mathrm{bp}$ shorter than the main allele peak. In this figure, (a) and (c) are homozygotes and (b), (d) and (e) are heterozygotes. 


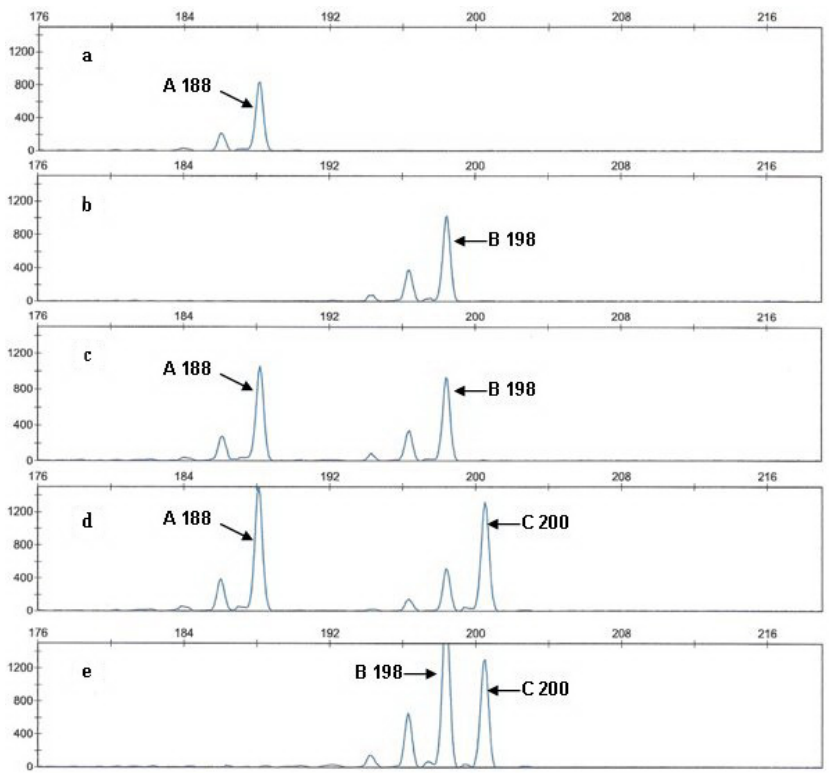

Figure 4. MNS64 locus with 3 alleles, A (188 bp), B (198 bp) and C (200 bp). All the alleles show well-resolved peaks. Allele A has a single stutter peak, 2 bp shorter than the main allele. Alleles B and C contain 2 stutters, 2 and 4 bp shorter than the main allele peaks, respectively. In this figure, (a) and (b) are homozygotes and (c), (d) and (e) are heterozygotes.
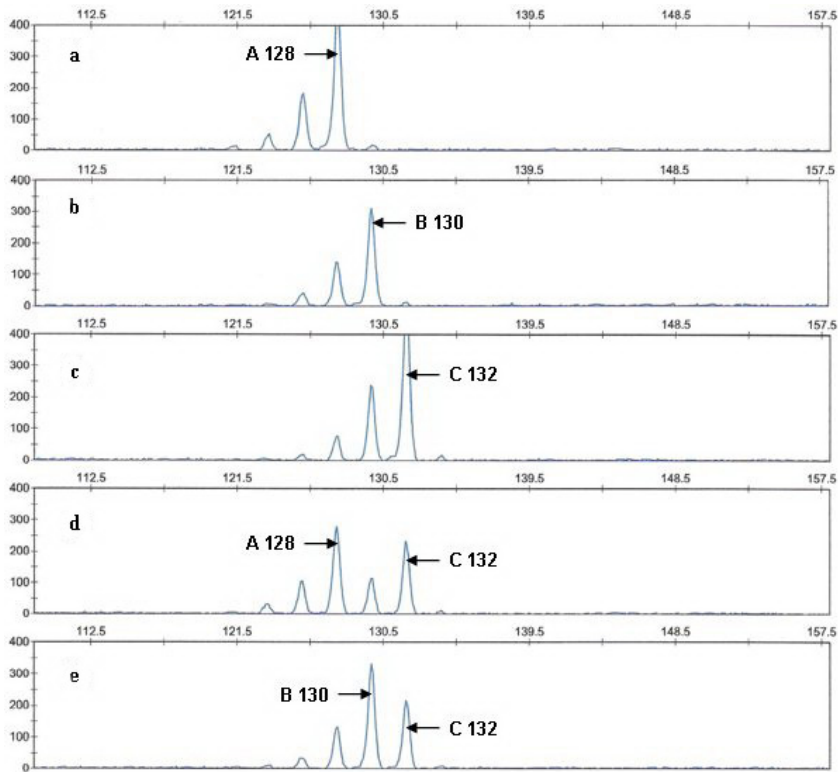

Figure 5. MB066 locus with 3 alleles, A (128 bp), B (130 bp) and C (132 bp). All the alleles show well-resolved peaks and also contain 2 stutters, 2 and 4 bp shorter than the main allele peaks, respectively. The homozygous presentation of alleles A and B is shown in (a) and (b), respectively. In the case of heterozygotes (d and e), the peak height of shorter allele is greater than the longer allele due to additive overlapping with stutter peaks. 


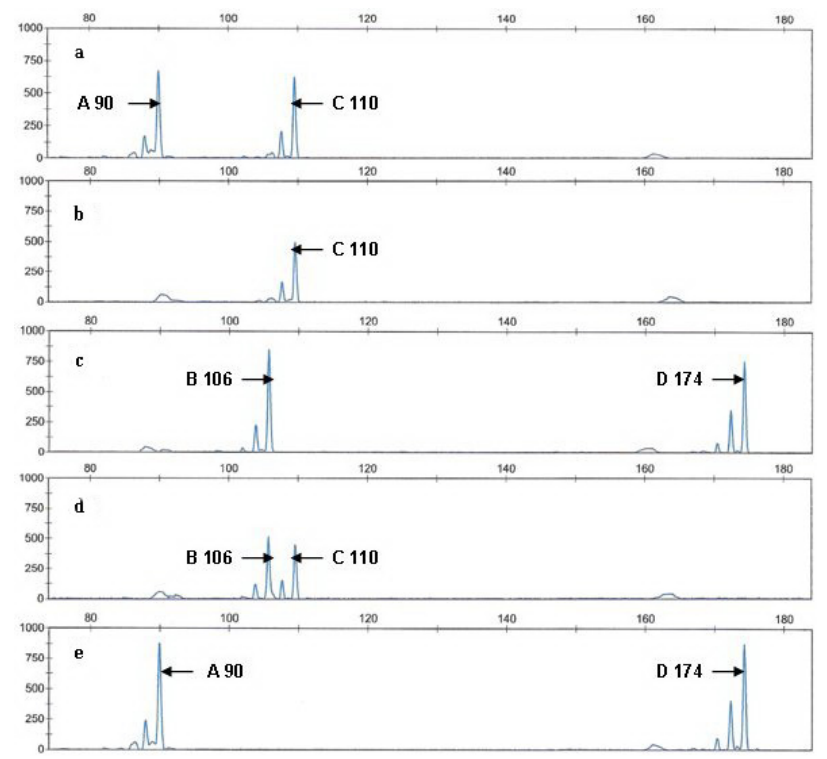

Figure 6. IOBT395 locus with 4 alleles, A(90 bp), B (106 bp), C (110 bp), and D (174 bp). All the alleles show well-resolved peaks. Since the allele sizes are not very close to each other there is no effect of stutters on heterozygote identification. In this figure, only (b) is a homozygote, whereas (a), (c), (d), and (e) are heterozygotes.

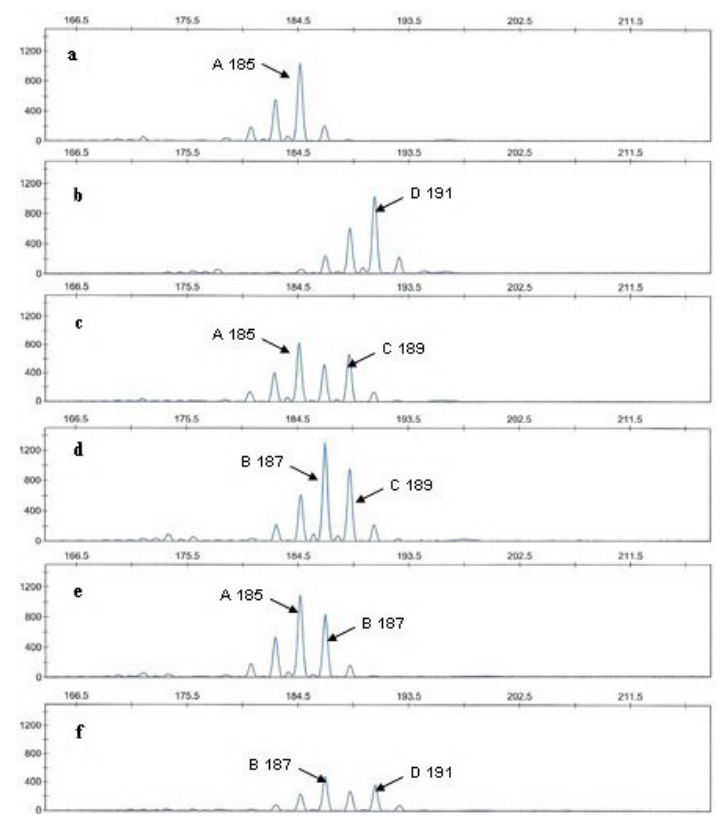

Figure 7. MCMAI locus with 4 alleles, A (185 bp), B (187 bp), C (189 bp), and D (191 bp). All the alleles show well-resolved peaks. However, due to the presence of sharp stutters as well as +2 peak in all the alleles the identification of genotypes is somewhat difficult. The difference between the peak heights of the two alleles in heterozygotes increases as the difference between their sizes decreases and vice versa. In this figure, (a) and (b) are homozygotes and (c), (d), (e), and (f) are heterozygotes. 
Both the alleles of RBP3 showed 1 stutter each (Figure 1), whereas 3 stutters were observed in the electrophoretogram of BM3501 though the -1 peak was very short (Figure 2). The alleles A and B of MCM 38 had 1 stutter each and the allele $C$ had 2 stutters (Figure 3 ). In the case of MNS64, the allele A showed a single stutter, whereas alleles B and C had 2 stutters each (Figure 4). All the three alleles of MB066 possessed 2 sharp stutters as shown in Figure 5. The 4 alleles of IOBT395 locus were different enough in size to completely negate the interference of stutters on allelic identification (Figure 6). All the alleles of MCMAI locus presented sharp stutters and a small +2 peak that might have some impact on the recognition of correct alleles (Figure 7). It has been suggested that the presence of stutter bands or peaks, generated by slippage of Taq polymerase during PCR, can make it difficult to score the alleles reliably (Harker, 2001; Johansson et al., 2003), especially when there are large signal intensity differences between alleles and/or the lengths of two alleles in a heterozygous individual differ by only a few nucleotides (Hoffman and Amos, 2005).

In conclusion, microsatellite markers are inevitable tools for molecular diversity analysis; however, correct identification of alleles in the presence of co-amplified nonspecific fragments is important for reliable interpretation of data.

\section{REFERENCES}

Banhos A, Hrbeck T, Gravena W, Sanaiotti T, et al. (2008). Genomic resources for the conservation and management of the harpy eagle (Harpia harpyja, Falconiformes, Accipitridae). Genet. Mol. Biol. 31: 146-154.

Bonin A, Bellemain E, Bronken EP, Pompanon F, et al. (2004). How to track and assess genotyping errors in population genetics studies. Mol. Ecol. 13: 3261-3273.

Chan CH, Zhao Y, Cheung MY and Chambers GK (2008). Isolation and characterization of microsatellites in the kakerori (Pomarea dimidiata) using feathers as source of DNA. Conserv. Genet. 9: 1067-1070.

Harker N (2001). Collection, Reporting and Storage of Microsatellite Genotype Data. In: Plant Genotyping: the DNA Fingerprinting of Plants (Henry RJ, ed.). CAB International, Wallingford, UK \& New York, USA, 251-264.

Hoffman JI and Amos W (2005). Microsatellite genotyping errors: detection approaches, common sources and consequences for paternal exclusion. Mol. Ecol. 14: 599-612.

Johansson A, Karlsson P and Gyllensten U (2003). A novel method for automatic genotyping of microsatellite markers based on parametric pattern recognition. Hum. Genet. 113: 316-324.

Kawka M, Horbanczuk JO, Sacharczuk M, Zieba G, et al. (2007). Genetic characteristics of the Ostrich population using molecular methods. Poult. Sci. 86: 277-281.

Li Y, Wongprasert K, Shekhar M, Ryan J, et al. (2007). Development of two microsatellite multiplex systems for black tiger shrimp Penaeus monodon and its application in genetic diversity study for two populations. Aquaculture 266: $279-288$.

MacHugh DE, Shriver MD, Loftus RT, Cunningham P, et al. (1997). Microsatellite DNA variation and the evolution, domestication and phylogeography of taurine and zebu cattle (Bos taurus and Bos indicus). Genetics 146: 1071-1086.

Ryberg WA, Fitzgerald LA, Honeycutt RL and Cathey JC (2002). Genetic relationships of American alligator populations distributed across different ecological and geographic scales. J. Exp. Zool. 294: 325-333.

Selkoe KA and Toonen RJ (2006). Microsatellites for ecologists: a practical guide to using and evaluating microsatellite markers. Ecol. Lett. 9: 615-629.

Zhou H, Li D, Zhang Y, Yang T, et al. (2007). Genetic diversity of microsatellite DNA loci of Tibetan antelope (Chiru, Pantholops hodgsonii) in Hoh Xil National Nature Reserve, Qinghai, China. J. Genet. Genomics 34: 600-607. 